\title{
Pharmacologic Considerations in Use and Development of Antituberculosis Drugs
}

\author{
Geraint Davies \\ Institutes of Infection and Global Health and of Translational Medicine, University of Liverpool, \\ Liverpool L69 7BE, United Kingdom \\ Correspondence: gdavies@liv.ac.uk
}

Rational development and deployment of antituberculosis drugs depend on a comprehensive understanding of the pharmacokinetics and pharmacodynamics that underlie their clinical behavior. Successful implementation of a pharmacokinetic-pharmacodynamic approach faces difficulties that, although not unique to tuberculosis as a therapeutic area, in combination pose a significant scientific challenge. In recent years, a multidisciplinary response combining new technological and analytical approaches has begun to directly address many of these issues, shedding light on some previously poorly understood aspects of drug distribution and response. These advances have important implications for optimization of existing and development of novel drug regimens, putting quantitative pharmacology at the heart of preclinical and early drug development.

$P_{\mathrm{t}}^{\mathrm{h}}$ harmacology is the key scientific discipline that underpins rational development and deployment of drugs, from the preclinical to postmarketing phases of the drug life cycle and across the dimensions of efficacy and toxicity. The pharmacological approach and attitude is generic, providing a framework within which the subject matter-specific elements of a therapeutic problem can be assembled, analyzed, and more completely understood. Implementing such an integrated approach in practice has always been challenging, however, and nowadays draws heavily on advanced quantitative approaches, such as mathematical and statistical modeling and systems biology. At the macro scale, these tools have been successfully applied to understanding real-life population variability in drug response, incorporating the insights of pharmacogenetics, and facilitating better predictions of dosing regimens and outcomes in clinical trials (Zhang et al. 2006). At the micro scale, the growing and fruitful interface between systems biology and pharmacology offers a new mode of incorporating the explosion of mechanistic information generated by postgenomic technologies into such models, capturing complicated or unexpected drug behavior that may otherwise appear perplexing (Mager et al. 2002; Vicini and van der Graaf 2013).

From a pharmacologic perspective, the therapy of tuberculosis (TB) presents numerous practical and intellectual challenges. From a pharmacokinetic (PK) standpoint the need for combination therapy with multiple agents, many of them prodrugs acting at clinically inaccessible sites on an organism with limited

Editors: Stefan H.E. Kaufmann, Eric J. Rubin, and Alimuddin Zumla

Additional Perspectives on Tuberculosis available at www.perspectivesinmedicine.org

Copyright (C) 2015 Cold Spring Harbor Laboratory Press; all rights reserved; doi: 10.1101/cshperspect.a021170

Cite this article as Cold Spring Harb Perspect Med 2015;5:a021170 
G. Davies

membrane permeability and potential for efflux, generates uncertainty about therapeutic drug concentrations at the site of action, which are usually the key drivers of drug action. From a pharmacodynamic (PD) perspective, added to the difficulty of selective toxicity when dealing with both host and pathogen is the repertoire of survival responses that Mycobacterium tuberculosis shows under drug pressure, which may be broadly characterized as resistance or persistence. These physiological changes have been noted in vitro and in animal models to result in complex, subpopulation- and drugspecific pharmacodynamic patterns, which are not easily captured by simple models (Davies 2010). However, despite these obvious problems, the value of a rigorous pharmacologically oriented approach to TB therapy has been convincingly shown in recent years, and developments in the field give grounds for increasing confidence that a more quantitative and integrated approach incorporating pharmacological concepts will become routine in the future (Davies and Nuermberger 2008). This article reviews these ideas and their application to date in tuberculosis with the hope that they will become better embedded across the range of disciplines that will be collectively required to make real improvements in tuberculosis treatment.

\section{GENERAL CONCEPTS}

Although of intrinsic interest in addressing the basic consideration of whether a compound is sufficiently drug-like to be useful in humans, the significance of PK parameters can only really be understood in terms of the relationship of drug concentrations to pharmacodynamics. A classic statement of unified and general PKPD principles was provided by Jusko et al. (1995) (Fig. 1). This schema captures the key elements of drug action and also allows for a more mechanistic perspective to develop from an initially more empirical approach in a way that can be applied to the preclinical and clinical contexts. Pharmacokinetics acts as the driver or "forcing function" of the PK-PD system, with the linkage to pharmacodynamics mediated by the binding affinity of the drug to the target, the properties of the target (biosignal), and the downstream consequences of target binding (transduction). The emphasis on distribution of drug to the "biophase" at the site of action and allowance for possible differences in the nature of and binding to the target are important features of this general model highly relevant to tuberculosis, although in some important cases these aspects may be as yet poorly characterized (Zhang and Mitchison 2003). In addition, the nature of the observed phenotypic

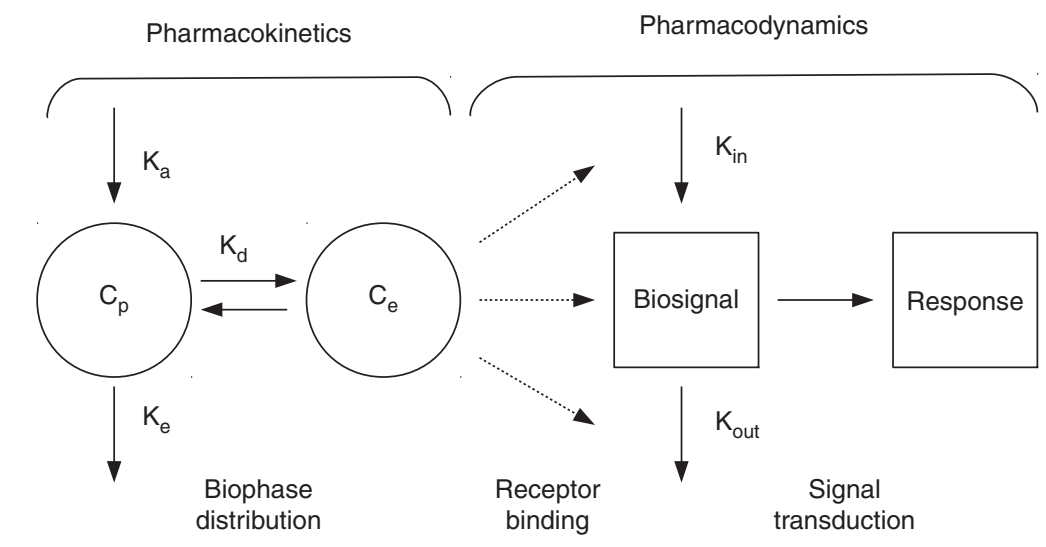

Figure 1. Components of pharmacokinetic-pharmacodynamic models. Determinants of drug action include pharmacokinetics (systemic absorption $\left[K_{a}\right]$ and elimination $\left[K_{e}\right]$ of drug distribution of a biophase $\left[K_{d}\right]$ ) and pharmacodynamics (direct binding to or inhibition or stimulation of production $\left[\mathrm{K}_{\mathrm{in}}\right]$ or removal $\left[\mathrm{K}_{\text {out }}\right]$ of a mediator biosignal, and transduction of the response). (Modified from Jusko et al. 1995.) 
response, typically inhibition of cell growth or cell death, and the precise molecular pathways that lead to it following binding of the drug to its target remain highly active research areas (Kohanski et al. 2010; Kim et al. 2013).

\section{PHARMACOKINETICS}

Pharmacokinetics deals with the mechanisms and variability of absorption, distribution, metabolism, and excretion of drugs. The PK properties of first- and second-line antituberculosis drugs are very diverse, reflecting almost the whole spectrum of physicochemical and metabolic possibilities (Global Alliance for TB Drug Development 2008; Koul et al. 2011). In fact, the current TB pharmacopoeia still includes drugs that proved to be of key historical importance in our understanding of the major routes of drug metabolism and interaction. Early studies of the wide clinical variability of elimination of isoniazid led to the recognition of the $\mathrm{N}$-acetylation pathway (Johnson 1954), whereas the serious drug interactions provoked by rifampicin helped to clarify the metabolic control of hepatic mixed function microsomal oxidases and interactions with drug transport mechanisms (Chen and Raymond 2006). This diversity of PK properties is important to consider in the construction of the combination regimens that are necessary for successful treatment of TB and in combining these with modern antiretroviral therapy in HIV-coinfected patients (Dooley et al. 2012). Some existing and new antituberculosis drugs such as rifapentine, clofazimine, bedaquiline, and delaminid have relatively undesirable PK properties such as bioavailability problems, very high protein binding and prolonged half-lives which may result in complex formulation, dosing, and coadministration issues. In extreme cases, the result may be a combination regimen composed of drugs that have poorly matched PK parameters. This situation may sometimes be of clinical importance. Rifapentine-based regimens, which rely on isoniazid as the sole companion drug in the continuation phase of treatment, are vulnerable to failure of treatment with the emergence of rifamycin monoresistance in $\mathrm{HIV}$-infected individ- uals (Vernon et al. 1999). In addition, the risk of failure may be related to acetylator status and consequently pharmacokinetic exposure to isoniazid (Weiner et al. 2003). This phenomenon appears to support the interpretation that it is mismatching of the elimination half-lives of the two drugs resulting in periods of functional monotherapy during treatment that promotes the acquisition of resistance. However, this phenomenon has also been observed with other rifamycins with less extreme mismatching (Nettles et al. 2004; Li et al. 2005; Weiner et al. 2005), and some recent in vitro experiments appear to challenge this concept as a general mechanism (Srivastava et al. 2011), suggesting that pharmacokinetics, as measured in plasma at least, may not be the only explanation. Variability of the PK parameters of particular drugs among individuals and its clinical consequences has long been debated among TB pharmacologists. Reports of very variable plasma concentrations from PK studies or therapeutic drug monitoring programs are common in the literature, although there is currently no global overview of the extent of this variability or its determinants (Peloquin et al. 1997; McIlleron et al. 2006; Njiland et al. 2006; Requena-Méndez et al. 2012). Such individual reports should be interpreted with some caution, because numerous factors including the details of the study protocol and bioanalytical methods may affect the estimates considerably, whereas in most cases the likely clinical impact is difficult to assess directly in terms of the PK-PD relationship. Most traditional pharmacokinetic studies were not explicitly designed to assess impact on efficacy, and only a few claim to have been able to show an impact on clinical outcomes (Chideya et al. 2009; Burhan et al. 2013). One recent such study, however, used classification and regression tree methods to identify critical thresholds for treatment success for each drug contained in the first-line regimen. It appeared that the greater the number of drugs in the regimen for which plasma concentrations fell below these thresholds, the higher was the risk of treatment failure or relapse (Pasipanodya et al. 2013). The investigators suggested that this could be interpreted as supporting the concept 
of interindividual variability in pharmacokinetics as the primary driver of ineffective therapy and of the development of resistance. However, the study was based on a relatively small number of patients with an unusually high rate of poor outcomes. Further studies of this kind using sparser sampling strategies on larger numbers of patients would be necessary to reliably validate these interesting results. Using a different approach, the only meta-analysis of published PK data attempted to date focused on studies that reported isonaizid acetylator status, concluding that in general rapid acetylator phenotype was associated with poorer outcomes ( $\mathrm{Pa}$ sipanodya et al. 2012).

Although plasma concentrations of drugs may in principle bear some useful relationship to drug efficacy, such a link is likely to be indirect. The usual site of disease in tuberculosis is the lung parenchyma, and the pathology of the disease results in a variety of physicochemical environments ranging from normal tissue to heavy inflammatory infiltrates to areas of dense, poorly vascularized caseous necrosis into which drug penetration is believed to be poor. Given the variety of properties of TB drugs, a number of which are weakly basic and/or prodrugs, intrapulmonary distribution is thus likely to be complex. Until recently little information was available on drug concentrations in the biophase at the site of action. Small bronchoscopy studies have evaluated the ratio of concentrations of first-line drugs at around the time of peak plasma concentrations in epithelial lining fluid and alveolar cells, concluding that in general drug penetration is adequate at these sites (Conte et al. 2001, 2002, 2004). Because the vast majority of Mycobacterium tuberculosis (MTB) organisms are, however, located in areas of diseased lung, these findings may not be very pertinent to the critical biophase concentration. Recently, use of ${ }^{11} \mathrm{C}$ labeled drugs and PET-CT (positron emission tomography-computed tomography) imaging in a primate model of disease has enabled a clearer picture to be obtained, suggesting that pulmonary uptake of first-line drugs results in tissue concentrations generally similar to or higher than that found in plasma and three- to 10-fold higher than the typical minimum inhibitory concentrations (Liu et al. 2010). An alternative approach has made use of a rabbit model of disease and postmortem tissue PK measurements to specifically study the distribution of first-line drugs and moxifloxacin into a variety of lesions as well as macroscopically normal lung tissue (Kjellsson et al. 2012). Using a modeling approach to analyze this relatively sparse data, the investigators were able to study the expected PK exposure in tissue relative to that in plasma. Interestingly, exposure achieved in lesions was lower than in plasma for most of the drugs studied, with the exception of moxifloxacin for which it was approximately twofold higher. Label-free MALDI (matrix-assisted laser desorption/ionization) mass spectrometric imaging has also been used to study the temporospatial distribution of moxifloxacin into pulmonary lesions in the same rabbit model (Prideaux et al. 2011). These data appear to confirm that moxifloxacin may accumulate homogenously within lesions with appreciable time delay in elimination, again generating at least twofold higher exposures overall, whereas the other drugs studied either did not penetrate the lesions or were equilibrated/eliminated much more rapidly. The methods described here remain to be fully developed but offer some hope that meaningful measurements of antituberculosis drugs in the biophase can be obtained, at least in animal models, and could in principle result in more precise estimates of the true $\mathrm{PK}-\mathrm{PD}$ relationship.

Developments in pharmacogenomic technologies have also been applied to the study of interindividual pharmacokinetic variability in TB. The paradigm case is isoniazid, for which extensive studies of polymorphism at the NAT-2 locus have been performed in various geographical populations. The frequency of mutant alleles is clearly a strong determinant of the distribution of isoniazid exposure in any given population, and this parameter has been directly linked to the early bactericidal activity (EBA) attributable to the drug (Donald et al. 2004). Variability in the pattern and diversity of PD response in the early days of therapy may therefore be strongly influenced by this locus. Rifampicin shows almost as much PK var- 
iability as isoniazid, but the explanation may be more complex and has been more difficult to link to pharmacodynamics. Rifampicin is a substrate and an inducer of numerous drug metabolizing enzymes and drug transporters (Williamson et al. 2013). Recently, the hepatic enzymes that may be responsible for its metabolism have been more clearly characterized with both a human hepatic carboxyesterase (CES1) (Zhu et al. 2008) and arylacetamide deacetylase (AADAC) possibly playing a significant role (Shimizu et al. 2012; Nakajima et al. 2011). Single-nucleotide polymorphisms (SNPs) are known to exist in both loci but have not yet been extensively studied in relation to rifampicin and other rifamycins. SNPs in the hepatic organic anion uptake transporter gene SLCO1B1 have also been linked to rifampicin exposure in two clinical studies (Weiner et al. 2010; Chigutsa et al. 2011). This transporter controls hepatic uptake of rifampicin and the relevant null alleles are widely distributed in African populations, perhaps providing a basis for the low exposures observed in some African PK studies. The interplay between the various possible determinants described here in the control of PK variability in rifamycins remains to be fully elucidated. Furthermore the complex and possibly bidirectional drug-drug interactions that can result mean that pharmacological considerations loom large in codevelopment of combined TB-HIV treatment strategies that include rifamycins (Dooley et al. 2012).

These problems of potentially unreliable or highly variable drug delivery have begun to be addressed by nanoformulation strategies with a focus on the inhalational route of administration. The possibility of simultaneously reducing systemic exposure and achieving higher and more sustained drug concentrations in the lungs and perhaps alveolar macrophages seems to promise reduced toxicity and greater efficacy, especially for those drugs with particularly poor physicochemical/PK properties and/or problematic toxicities such as the injectables currently required for treatment of multidrug-resistant (MDR) TB. Recent studies suggest that a dry powder porous nanoparticle-aggregate particle (PNAP) format may be well suited for this purpose and the technology has been successfully applied to capreomycin, rifampicin, and PA-824 in animal models (Garcia-Contreras et al. 2007; Sung et al. 2009a,b). A number of other promising nanoformulation approaches are also available for this purpose (Pandey et al. 2003; Zahoor et al. 2005), whereas other groups have instead used subcutaneous or intravenous liposomal or niosomal formulations to target the pulmonary compartment (Adams et al. 1999; El-Ridy et al. 2007). Alternatively, using nanodispersion or nanoparticle techniques it may be possible to obtain oral formulations of drugs previously only delivered by injection (Pandey and Khuller 2007). Although much work remains to be performed in this area, these technologies have considerable potential to overcome some of the current limitations of drug delivery in tuberculosis.

\section{PHARMACODYNAMICS}

Traditionally the study of pharmacodynamics in tuberculosis in vitro and in vivo has leaned heavily on the measurement of inhibition of growth of MTB organisms. From a practical perspective this is simpler and less time-consuming than attempting to measure definitive killing, but it raises the important issue of whether assays and clinical methods in current use faithfully represent the kind of PD activity required for long-term cure of the disease. The range of end points used in clinical trials suggests that a useful distinction should be drawn between drugs that effectively reduce bacillary load during the early phases of treatment, preventing death and the emergence of resistance, and those that appear to have their critical activity later on and that prevent treatment failure and relapse (Lienhardt and Davies 2010). This broad clinical distinction between "bactericidal activity" and "sterilizing activity" has been increasingly interpreted in terms of drug action against qualitatively different subpopulations of organisms, a hypothesis first put forward by Canetti (1962) and Mitchison (1979). Although the precise defining characteristics of these subpopulations of organisms remain an open question, most researchers now recog- 
G. Davies

nize that assays directed at log-phase in vitro organisms alone are likely to be an unreliable tool for drug screening and development and that assays that incorporate at least some of the conditions pertaining in the host such as hypoxia, low $\mathrm{PH}$, and alternative carbon sources are essential (Franzblau et al. 2012). Another important development has been the development and adaptation to tuberculosis of in vitro systems that can simulate dynamic PK-like patterns of drug exposure rather than the static concentrations typically used. Such systems are able to assist in defining the nature of the PKPD relationship in relation to efficacy and resistance (Gumbo et al. 2004, 2007) and have been implemented using altered growth conditions, which may relate to in vivo physiological states (Gumbo et al. 2009). Recent work has resulted in altered recommendations for susceptibility testing and dosing (Gumbo 2010; Srivastava et al. 2011). Whether and which of these assays correlate with "sterilizing activity" remain unknown, however. In the clinical context, methods to directly differentiate between the qualitatively different states of MTB observed in vitro are in their infancy, although there have been some advances in recent years. Several different methods of measuring bacillary load in clinical specimens are now in use, which may infor- mally represent these different states enabling more precise evaluation of drug activity against them in clinical trials. In addition to colony counting on solid media, liquid culture systems are now being increasingly deployed in clinical trial laboratories (Diacon et al. 2010b), whereas molecular methods such as Xpert (Kayigire et al. 2013) and other real-time polymerase chain reaction (PCR) methods directed at various mRNA or rRNA targets (Honeyborne et al. 2011) can in principle give an estimate of bacillary load that is less dependent on the growth characteristics of those organisms. Most recently, the discovery of several resuscitation promotion factors in MTB has enabled development of a method of enhancing liquid culture, apparently also revealing organisms not quantified by routine methods (Mukamolova et al. 2009). These new technologies raise the possibility of a more sophisticated PD model than is currently in use, which could be more reliable in terms of interpretation (Fig. 2) (Davies 2010). This interplay between our increasing understanding of mycobacterial physiology and drug action in vitro and the laboratory and analytical methods used in early phase clinical trials is absolutely essential to improve our ability to differentiate those drugs that are truly sterilizing from those that simply reduce bacillary load.

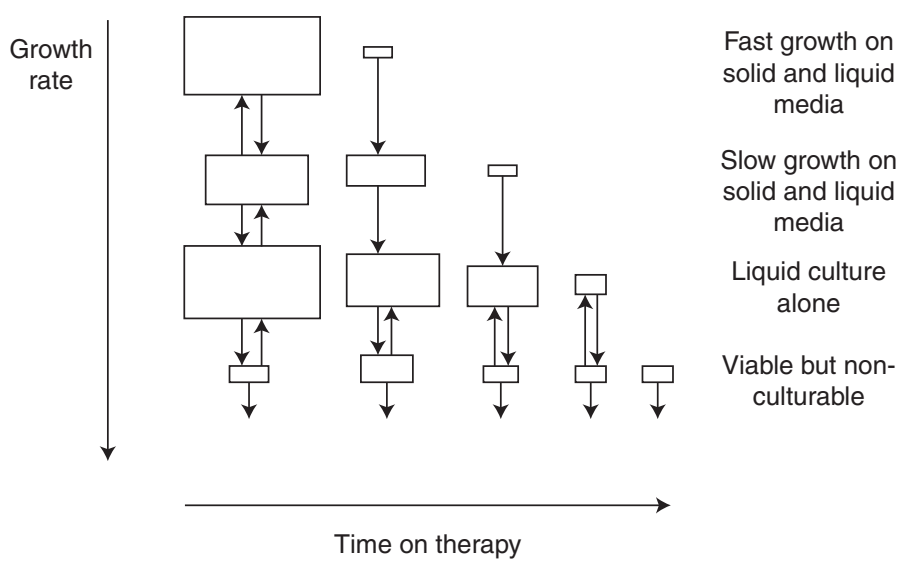

Figure 2. Simplified scheme of a transition pharmacodynamic model for tuberculosis treatment. Boxes represent the size of subpopulations with arrows representing possible transitions between them. Each subpopulation has a defined death rate, which has been omitted for clarity, with the exception of the viable but nonculturable population at the bottom of the figure. As therapy proceeds, subpopulations are sequentially eliminated according to their characteristics. 
Currently all clinical drug development programs include a small, usually dose-ranging study of EBA. Although the possible problems of interpretation of such studies have been outlined above, historical EBA studies have been important because they established the possibility of PK-PD analysis in tuberculosis, where previously the development process had been much more informal and heavily dependent on animal experiments (Donald et al. 2003). The paradigm case of isoniazid was the first time that the dose-response and concentration-response relationships incorporating pharmacogenetic variability had been fully defined in humans for any tuberculosis drug (Donald et al. 1997, 2004). The idea was successfully applied to other classes of drugs including rifamycins and fluoroquinolones (Sirgel et al. 2005; Johnson et al. 2006; Diacon et al. 2007). These efforts have been of particular interest because they have shown that changes in physicochemical properties and intrinsic activity among members of each class may markedly affect the EBA. In particular, recognition that the dose-response curves for rifampicin and rifapentine remained incomplete as measured by EBA and that AUC/MIC (area under the curve/minimum inhibitory concentration) ratios achieved in humans compared unfavorably with those achieved in animals, led to new efforts to repurpose and optimize these drugs using modern PK-PD methods. The advent of completely new classes of drugs has also been instructive. The two drugs recently licensed for MDR TB, bedaquiline and delaminid, both show EBA that appears poor by comparison with isoniazid (Rustomjee et al. 2008a; Diacon et al. 2011), whereas clearly adding important activity in the context of MDR TB regimens (Diacon et al. 2009; Gler et al. 2012). More important in the case of bedaquiline, the pattern of pharmacodynamic response reflects the mechanism of action of the drug (depletion of ATP) and is time dependent, creating a mirror image of the response expected based on repeated studies of control regimens based on isoniazid (Diacon et al. 2013). Finally, dose-ranging EBA studies for PA-824 were repeated when EBA was initially observed to be low and not increas- ing with dose (Diacon et al. 2010a, 2012a). In fact, PA-824 monotherapy outperformed a standard multidrug control regimen, and the lack of dose-response was attributable to selection of dose ranges that animal studies suggested exceeded the maximum response. These new findings argue that although useful information can be derived from EBA studies, they must be carefully interpreted in the context of existing knowledge of the pharmacology of the drug and relevant preclinical findings.

Although the definitive measure of "sterilizing activity" remains relapse of disease after treatment is stopped, often combined with treatment failure at the end of treatment, several groups have attempted to extend the quantitative EBA approach using longer periods of sampling during the first three months when bacteriological measures are still available to provide information on response. Although culture conversion at fixed time points such as two months is currently regarded as the best available candidate surrogate end point for treatment failure/ relapse, this is largely because it is supported by historical experience (Food and Drug Administration 2013). The reasonable predictive performance of two-month culture as assessed over these trials (Phillips et al. 2013; Wallis et al. 2013 ) is undermined by its inflexibility and relative lack of power. To fully capitalize on information from early bacteriological biomarkers, it has been necessary to adopt a more sophisticated statistical modeling approach, of a type more familiar to PK-PD scientists than clinical trialists. Using the full profile of repeated bacteriological measurements during treatment it is possible to get a fuller appreciation of the progress of treatment and perhaps more importantly the pattern of PD response. Initial empirical approaches based on colony counting data from current first-line regimens described a characteristically biphasic PD pattern with the elimination rate of sputum organisms (Davies et al. 2006; Rustomjee et al. 2008b). However, novel regimens have presented diverse patterns of response, necessitating more flexible approaches capable of capturing and yet comparing these qualitatively different profiles (Diacon et al. 2012b). More recently, simple models of elimi- 
G. Davies

nation fitted to liquid culture data have been developed (Martineau et al. 2011; Chigutsa et al. 2013). Changes in time to positivity in MGIT (Mycobacteria Growth Indicator Tube) appear to be similar in magnitude and variability to those observed with colony counting (Diacon et al. 2010b). An appropriate model for these data appears, however, to be monophasic, perhaps related to decontamination of specimens during laboratory processing. Changes in elimination rate in such models appear capable of predicting long-term outcome in individual patients (Sloan et al. 2013). Finally, a similar approach has been used to model 16S rRNA measurements (Honeyborne et al. 2011). These modeling approaches to early phase clinical trials are gradually gaining wider acceptance and promise to revolutionize the way that early phase development of combination regimens is performed in tuberculosis (Diacon et al. 2012b). However, larger cohorts with appropriate long-term follow-up and relating to a broader range of drug regimens will be needed to establish their reliability in relation to the twomonth culture conversion end point.

\section{SYSTEMS PHARMACOLOGY}

The emerging discipline of systems pharmacology aims to harness the wealth of new information made available from postgenomic technologies to understand mechanisms of drug response and toxicity in greater detail. A number of groups have developed systems models incorporating the recent explosion of molecular data from MTB, but they have so far largely been used to deepen our understanding of global cellular processes under different growth conditions (Beste and McFadden 2010; Fang et al. 2010; Galagan et al. 2013). However, such models are ideal tools to study the perturbations induced by drug pressure and the robustness of the whole organism or particular physiological pathways to their ramifications, such as postantibiotic effect and cell death (Raman et al. 2009; Fang et al. 2011). In addition, the possibility of representing critical parts of these system models as a subsystem of a multiscale PK-PD model (Goutelle et al. 2011) may be important for drug regimens with specific or unusual mechanisms of action, the results of which could be measured in vivo (e.g., bedaquiline). Incorporation of such information may be of overriding importance in correctly understanding what it is that generates empirically different patterns of $\mathrm{PD}$ response in vitro or in vivo and what the implications are for clinical outcome.

\section{CONCLUDING REMARKS}

Improving how antituberculosis drugs are used and developed depends on a complete understanding of their pharmacology, construed in the broadest possible sense. Although tuberculosis poses some specific challenges, advances in recent years have resulted in a fruitful multidisciplinary response that has significantly changed our understanding of the therapeutics of the disease and how best to develop new combinations of drugs. It is only by intelligently integrating and using information relating to all aspects of the PK-PD relationship that important biological and clinical phenomena can be usefully incorporated and their implications for drug development and deployment clearly understood. The pharmacological concepts and tools described here are an effective means of promoting that understanding across the diverse disciplines that will contribute to better future treatments for tuberculosis.

\section{REFERENCES}

Adams LB, Sinha I, Franzblau SG, Krahenbuhl JL, Mehta RT. 1999. Effective treatment of acute and chronic murine tuberculosis with liposome-encapsulated clofazimine. Antimicrob Agents Chemother 43: 1638-1643.

Beste DJV, McFadden J. 2010. System-level strategies for studying the metabolism of Mycobacterium tuberculosis. Mol Biosyst 6: 2363-2372.

Burhan E, Ruesen C, Ruslami R, Ginanjar A, Mangunnegoro H, Ascobat P, Donders R, van Crevel R, Aarnoutse R. 2013. Isoniazid, rifampin, and pyrazinamide plasma concentrations in relation to treatment response in Indonesian pulmonary tuberculosis patients. Antimicrob Agents Chemother 57: 3614-3619.

Canetti G. 1962. The eradication of tuberculosis: Theoretical problems and practical solutions. Tubercle 43: 301321.

Chen J, Raymond K. 2006. Roles of rifampicin in drug-drug interactions: Underlying molecular mechanisms involv- 
Pharmacological Considerations in Tuberculosis

ing the nuclear pregnane X receptor. Ann Clin Microbiol Antimicrob 5: 3.

Chideya S, Winston CA, Peloquin CA, Bradford WZ, Hopewell PC, Wells CD, Reingold AL, Kenyon TA, Moeti TL, Tappero JW. 2009. Isoniazid, rifampin, ethambutol, and pyrazinamide pharmacokinetics and treatment outcomes among a predominantly HIV-infected cohort of adults with tuberculosis from Botswana. Clin Infect Dis 48: $1685-1694$.

Chigutsa E, Visser ME, Swart EC, Denti P, Pushpakom S, Egan D, Holford NHG, Smith PJ, Maartens G, Owen A, et al. 2011. The SLCO1B1 rs4149032 polymorphism is highly prevalent in South Africans and is associated with reduced rifampin concentrations: Dosing implications. Antimicrob Agents Chemother 55: 4122-4127.

Chigutsa E, Patel K, Denti P, Visser M, Maartens G, Kirkpatrick CMJ, McIlleron H, Karlsson MO. 2013. A timeto-event pharmacodynamic model describing treatment response in patients with pulmonary tuberculosis using days to positivity in automated liquid mycobacterial culture. Antimicrob Agents Chemother 57: 789-795.

Conte JE Jr, Golden JA, Kipps J, Lin ET, Zurlinden E. 2001. Effects of AIDS and gender on steady-state plasma and intrapulmonary ethambutol concentrations. Antimicrob Agents Chemother 45: 2891-2896.

Conte JE, Golden JA, McQuitty M, Kipps J, Duncan S, McKenna E, Zurlinden E. 2002. Effects of gender, AIDS, acetylator status on intrapulmonary concentrations of isoniazid. Antimicrob Agents Chemother 46: 2358-2364.

Conte JE, Golden JA, Kipps JE, Lin ET, Zurlinden E. 2004. Effect of sex and AIDS status on the plasma and intrapulmonary pharmacokinetics of rifampicin. Clin Pharmacokinet 43: 395-404.

Davies GR. 2010. Early clinical development of anti-tuberculosis drugs: Science, statistics and sterilizing activity. Tuberc Edinb Scotl 90: 171-176.

Davies GR, Nuermberger EL. 2008. Pharmacokinetics and pharmacodynamics in the development of anti-tuberculosis drugs. Tuberculosis (Edinb) 88: S65-S74.

Davies GR, Brindle R, Khoo SH, Aarons LJ. 2006. Use of nonlinear mixed-effects analysis for improved precision of early pharmacodynamic measures in tuberculosis treatment. Antimicrob Agents Chemother 50: 3154-3156.

Diacon AH, Patientia RF, Venter A, van Helden PD, Smith PJ, McIlleron H, Maritz JS, Donald PR. 2007. Early bactericidal activity of high-dose rifampin in patients with pulmonary tuberculosis evidenced by positive sputum smears. Antimicrob Agents Chemother 51: 2994-2996.

Diacon AH, Pym A, Grobusch M, Patientia R, Rustomjee R, Page-Shipp L, Pistorius C, Krause R, Bogoshi M, Churchyard G, et al. 2009. The diarylquinoline TMC207 for multidrug-resistant tuberculosis. $N$ Engl J Med 360: 2397-2405.

Diacon AH, Dawson R, Hanekom M, Narunsky K, Maritz SJ, Venter A, Donald PR, van Niekerk C, Whitney K, Rouse DJ, et al. 2010a. Early bactericidal activity and pharmacokinetics of PA-824 in smear-positive tuberculosis patients. Antimicrob Agents Chemother 54: 3402-3407.

Diacon AH, Maritz JS, Venter A, van Helden PD, Andries K, McNeeley DF, Donald PR. 2010b. Time to detection of the growth of Mycobacterium tuberculosis in MGIT 960 for determining the early bactericidal activity of antitu- berculosis agents. Eur J Clin Microbiol Infect Dis 29: $1561-1565$.

Diacon AH, Dawson R, Hanekom M, Narunsky K, Venter A, Hittel N, Geiter LJ, Wells CD, Paccaly AJ, Donald PR. 2011. Early bactericidal activity of delamanid (OPC67683) in smear-positive pulmonary tuberculosis patients. Int J Tuberc Lung Dis 15: 949-954.

Diacon AH, Dawson R, du Bois J, Narunsky K, Venter A, Donald PR, van Niekerk C, Erondu N, Ginsberg AM, Becker P, et al. 2012a. Phase II dose-ranging trial of the early bactericidal activity of PA-824. Antimicrob Agents Chemother 56: 3027-3031.

Diacon AH, Dawson R, von Groote-Bidlingmaier F, Symons G, Venter A, Donald PR, van Niekerk C, Everitt D, Winter H, Becker P, et al. 2012b. 14-day bactericidal activity of PA-824, bedaquiline, pyrazinamide, and moxifloxacin combinations: A randomised trial. Lancet 380: 986-993.

Diacon AH, Dawson R, Von Groote-Bidlingmaier F, Symons G, Venter A, Donald PR, Conradie A, Erondu N, Ginsberg AM, Egizi E, et al. 2013. Randomized dose-ranging study of the 14-day early bactericidal activity of bedaquiline (TMC207) in patients with sputum microscopy smear-positive pulmonary tuberculosis. Antimicrob Agents Chemother 57: 2199-2203.

Donald P, Sirgel F, Botha F, Seifart H, Parkin D, Vandenplas M. 1997. The early bactericidal activity of isoniazid related to its dose size in pulmonary tuberculosis. Am J Respir Crit Care Med 156: 895-900.

Donald P, Sirgel F, Venter A, Parkin D, Seifart H, van de Wal B, Maritz J, Fourie P. 2003. Early bactericidal activity of antituberculosis agents. Expert Rev Anti Infect Ther 1: 141-155.

Donald P, Sirgel F, Venter A, Parkin D, Seifart H, van de Wal B, Werely C, van Helden P, Maritz J. 2004. The influence of human $\mathrm{N}$-acetyltransferase genotype on the early bactericidal activity of isoniazid. Clin Infect Dis 39: 1425-1430.

Dooley KE, Kim PS, Williams SD, Hafner R. 2012. TB and HIV therapeutics: Pharmacology research priorities. AIDS Res Treat 2012: 1-9.

El-Ridy MS, Mostafa DM, Shehab A, Nasr EA, Abd El-Alim S. 2007. Biological evaluation of pyrazinamide liposomes for treatment of Mycobacterium tuberculosis. Int J Pharm 330: $82-88$

Fang X, Wallqvist A, Reifman J. 2010. Development and analysis of an in vivo-compatible metabolic network of Mycobacterium tuberculosis. BMC Syst Biol 4: 160.

Fang X, Wallqvist A, Reifman J. 2011. Modeling synergistic drug inhibition of Mycobacterium tuberculosis growth in murine macrophages. Mol Biosyst 7: 2622-2636.

Food and Drug Administration. 2013. Guidance for industry. Pulmonary tuberculosis: Developing drugs for treatment, Draft Guidance. FDA, Silver Spring, MD.

Franzblau SG, DeGroote MA, Cho SH, Andries K, Nuermberger E, Orme IM, Mdluli K, Angulo-Barturen I, Dick T, Dartois V, et al. 2012. Comprehensive analysis of methods used for the evaluation of compounds against Mycobacterium tuberculosis. Tuberculosis (Edinb) 92: 453-488.

Galagan JE, Minch K, Peterson M, Lyubetskaya A, Azizi E, Sweet L, Gomes A, Rustad T, Dolganov G, Glotova I, et al. 2013. The Mycobacterium tuberculosis regulatory network and hypoxia. Nature 499: 178-183. 
G. Davies

Garcia-Contreras L, Fiegel J, Telko MJ, Elbert K, Hawi A, Thomas M, VerBerkmoes J, Germishuizen WA, Fourie PB, Hickey AJ, et al. 2007. Inhaled large porous particles of capreomycin for treatment of tuberculosis in a guinea pig model. Antimicrob Agents Chemother 51: 2830-2836.

Gler MT, Skripconoka V, Sanchez-Garavito E, Xiao H, Cabrera-Rivero JL, Vargas-Vasquez DE, Gao M, Awad M, Park S-K, Shim TS, et al. 2012. Delamanid for multidrug-resistant pulmonary tuberculosis. $N$ Engl J Med 366: $2151-2160$.

Global Alliance for TB Drug Development. 2008. Handbook of anti-tuberculosis agents. Tuberculosis (Edinb) 88: 1169.

Goutelle S, Bourguignon L, Jelliffe RW, Conte JE Jr, Maire P. 2011. Mathematical modeling of pulmonary tuberculosis therapy: Insights from a prototype model with rifampin. J Theor Biol 282: 80-92.

Gumbo T. 2010. New susceptibility breakpoints for first-line antituberculosis drugs based on antimicrobial pharmacokinetic/pharmacodynamic science and population pharmacokinetic variability. Antimicrob Agents Chemother 54: 1484-1491.

Gumbo T, Louie A, Deziel M, Parsons L, Salfinger M, Drusano G. 2004. Selection of a moxifloxacin dose that suppresses drug resistance in Mycobacterium tuberculosis by use of an in vitro pharmacodynamic infection model and mathematical modelling. J Infect Dis 190: 1642-1651.

Gumbo T, Louie A, Liu W, Ambrose P, Bhavnani S, Brown D, Drusano G. 2007. Isoniazid's bactericidal activity ceases because of the emergence of resistance, not depletion of Mycobacterium tuberculosis in the log phase of growth. J Infect Dis 195: 194-201.

Gumbo T, Dona CSWS, Meek C, Leff R. 2009. Pharmacokinetics-pharmacodynamics of pyrazinamide in a novel in vitro model of tuberculosis for sterilizing effect: A paradigm for faster assessment of new antituberculosis drugs. Antimicrob Agents Chemother 53: 3197-3204.

Honeyborne I, McHugh TD, Phillips PPJ, Bannoo S, Bateson A, Carroll N, Perrin FM, Ronacher K, Wright L, van Helden PD, et al. 2011. Molecular bacterial load assay, a culture-free biomarker for rapid and accurate quantification of sputum Mycobacterium tuberculosis bacillary load during treatment. J Clin Microbiol 49: 3905-3911.

Johnson W. 1954. Biological acetylation of isoniazid. Nature 174: 744-745.

Johnson JL, Hadad DJ, Boom WH, Daley CL, Peloquin CA, Eisenach KD, Jankus DD, Debanne SM, Charlebois ED, Maciel E, et al. 2006. Early and extended early bactericidal activity of levofloxacin, gatifloxacin and moxifloxacin in pulmonary tuberculosis. Int J Tuberc Lung Dis 10: 605612.

Jusko WJ, Ko HC, Ebling WF. 1995. Convergence of direct and indirect pharmacodynamic response models. J Pharmacokinet Biopharm 23: 5-8; discussion 9-10.

Kayigire XA, Friedrich SO, Venter A, Dawson R, Gillespie SH, Boeree MJ, Heinrich N, Hoelscher M, Diacon AH, Pan African Consortium for the Evaluation of Anti-tuberculosis Antibiotics. 2013. Direct comparison of Xpert MTB/RIF assay with liquid and solid mycobacterial culture for quantification of early bactericidal activity. J Clin Microbiol 51: 1894-1898.
Kim J-H, O’Brien KM, Sharma R, Boshoff HIM, Rehren G, Chakraborty S, Wallach JB, Monteleone M, Wilson DJ, Aldrich CC, et al. 2013. A genetic strategy to identify targets for the development of drugs that prevent bacterial persistence. Proc Natl Acad Sci 110: 19095-19100.

Kjellsson MC, Via LE, Goh A, Weiner D, Low KM, Kern S, Pillai G, Barry CE 3rd, Dartois V. 2012. Pharmacokinetic evaluation of the penetration of antituberculosis agents in rabbit pulmonary lesions. Antimicrob Agents Chemother 56: $446-457$.

Kohanski MA, DePristo MA, Collins JJ. 2010. Sublethal antibiotic treatment leads to multidrug resistance via radical-induced mutagenesis. Mol Cell 37: 311-320.

Koul A, Arnoult E, Lounis N, Guillemont J, Andries K. 2011. The challenge of new drug discovery for tuberculosis. Nature 469: 483-490.

Lienhardt C, Davies G. 2010. Methodological issues in the design of clinical trials for the treatment of multidrugresistant tuberculosis: Challenges and opportunities. Int J Tuberc Lung Dis 14: 528-537.

Li J, Munsiff S, Driver C, Sackoff J. 2005. Relapse and acquired rifampin resistance in HIV-infected patients with tuberculosis treated with rifampin- or rifbutin-based regimens in New York City, 1997-2000. Clin Infect Dis 41: 83-91.

Liu L, Xu Y, Shea C, Fowler JS, Hooker JM, Tonge PJ. 2010. Radiosynthesis and bioimaging of the tuberculosis chemotherapeutics isoniazid, rifampicin and pyrazinamide in baboons. J Med Chem 53: 2882-2891.

Mager D, Wyska E, Jusko W. 2002. Diversity of mechanismbased pharmacodynamic models. Drug Metab Dispos 31: 510-518.

Martineau AR, Timms PM, Bothamley GH, Hanifa Y, Islam K, Claxton AP, Packe GE, Moore-Gillon JC, Darmalingam M, Davidson RN, et al. 2011. High-dose vitamin $\mathrm{D}_{3}$ during intensive-phase antimicrobial treatment of pulmonary tuberculosis: A double-blind randomised controlled trial. Lancet 377: 242-250.

McIlleron H, Wash P, Burger A, Norman J, Folb P, Smith P. 2006. Determinants of rifampicin, isoniazid, pyrazinamide and ethambutol pharmacokinetics in a cohort of tuberculosis patients. Antimicrob Agents Chemother 50: 1170-1177.

Mitchison D. 1979. Basic mechanisms of chemotherapy. Chest 76: 771-781.

Mukamolova GV, Turapov O, Malkin J, Woltmann G, Barer MR. 2009. Resuscitation promoting factors reveal an occult population of tubercle bacilli in sputum. Am J Respir Crit Care Med http://www.ncbi.nlm.nih.gov/pubmed/ 19875686 (Accessed November 27, 2009).

Nakajima A, Fukami T, Kobayashi Y, Watanabe A, Nakajima M, Yokoi T. 2011. Human arylacetamide deacetylase is responsible for deacetylation of rifamycins: Rifampicin, rifabutin, and rifapentine. Biochem Pharmacol 82: 1747 1756.

Nettles R, Mazo D, Alwood K, Gachuchi R, Maltas G, Wendel K, Cronin W, Hooper N, Bishai W, Sterling T. 2004. Risk factors for relapse and acquired rifamycin resistance after directly observed tuberculosis treatment: A comparison by HIV serostatus and rifamycin use. Clin Infect Dis 38: 731-736. 
Njiland H, Ruslami R, Stalenhoef J, Nelwan E, Alisjahbana B, Nelwan R, van der Ven A, Danusantoso H, Aarnoutse R, van Crevel R. 2006. Exposure to rifampicin is strongly reduced in patients with tuberculosis and type 2 diabetes. Clin Infect Dis 43: 848-854.

Pandey R, Khuller GK. 2007. Nanoparticle-based oral drug delivery system for an injectable antibiotic-Streptomycin. Evaluation in a murine tuberculosis model. Chemotherapy 53: 437-441.

Pandey R, Sharma A, Zahoor A, Sharma S, Khuller GK, Prasad B. 2003. Poly (DL-lactide-co-glycolide) nanoparticle-based inhalable sustained drug delivery system for experimental tuberculosis. J Antimicrob Chemother 52: 981-986.

Pasipanodya JG, Srivastava S, Gumbo T. 2012. Meta-analysis of clinical studies supports the pharmacokinetic variability hypothesis for acquired drug resistance and failure of antituberculosis therapy. Clin Infect Dis 55: $169-177$.

Pasipanodya JG, McIlleron H, Burger A, Wash PA, Smith P, Gumbo T. 2013. Serum drug concentrations predictive of pulmonary tuberculosis outcomes. J Infect Dis 208: 1464-1473.

Peloquin CA, Jaresko GS, Yong CL, Keung AC, Bulpitt AE, Jelliffe RW. 1997. Population pharmacokinetic modeling of isoniazid, rifampin, and pyrazinamide. Antimicrob Agents Chemother 41: 2670-2679.

Phillips PPJ, Fielding K, Nunn AJ. 2013. An evaluation of culture results during treatment for tuberculosis as surrogate endpoints for treatment failure and relapse. PloS ONE 8: e63840.

Prideaux B, Dartois V, Staab D, Weiner DM, Goh A, Via LE, Barry CE 3rd, Stoeckli M. 2011. High-sensitivity MALDI-MRM-MS imaging of moxifloxacin distribution in tuberculosis-infected rabbit lungs and granulomatous lesions. Anal Chem 83: 2112-2118.

Raman K, Vashisht R, Chandra N. 2009. Strategies for efficient disruption of metabolism in Mycobacterium tuberculosis from network analysis. Mol Biosyst 5: $1740-$ 1751.

Requena-Méndez A, Davies G, Ardrey A, Jave O, LópezRomero SL, Ward SA, Moore DAJ. 2012. Pharmacokinetics of rifampin in Peruvian tuberculosis patients with and without comorbid diabetes or HIV. Antimicrob Agents Chemother 56: 2357-2363.

Rustomjee R, Diacon AH, Allen J, Venter A, Reddy C, Patientia RF, Mthiyane TCP, De Marez T, van Heeswijk R, Kerstens R, et al. 2008a. Early bactericidal activity and pharmacokinetics of the diarylquinoline TMC207 in treatment of pulmonary tuberculosis. Antimicrob Agents Chemother 52: 2831-2835.

Rustomjee R, Lienhardt C, Kanyok T, Davies GR, Levin J, Mthiyane T, Reddy C, Sturm AW, Sirgel FA, Allen J, et al. 2008b. A Phase II study of the sterilising activities of ofloxacin, gatifloxacin and moxifloxacin in pulmonary tuberculosis. Int J Tuberc Lung Dis 12: 128 138.

Shimizu M, Fukami T, Kobayashi Y, Takamiya M, Aoki Y, Nakajima M, Yokoi T. 2012. A novel polymorphic allele of human arylacetamide deacetylase leads to decreased enzyme activity. Drug Metab Dispos 40: 1183-1190.
Pharmacological Considerations in Tuberculosis

Sirgel FA, Fourie PB, Donald PR, Padayatchi N, Rustomjee R, Levin J, Roscigno G, Norman J, McIlleron H, Mitchison DA. 2005. The early bactericidal activities of rifampin and rifapentine in pulmonary tuberculosis. Am J Respir Crit Care Med 172: 128-135.

Sloan D, Mwandumba H, Guwende C, Chipungu G, Butterworth A, Dutton J, Heyderman R, Corbett E, Khoo S, Davies G. 2013. Intensive phase bacillary elimination predicts clinical outcome in $\mathrm{HIV}^{+}$and $\mathrm{HIV}^{-}$Malawian adults with smear positive pulmonary TB. In Conference on Retroviruses and Opportunistic Infections 2013, Atlanta, GA. Abstract 858.

Srivastava S, Sherman C, Meek C, Leff R, Gumbo T. 2011. Pharmacokinetic mismatch does not lead to emergence of isoniazid- or rifampin-resistant Mycobacterium tuberculosis but to better antimicrobial effect: A new paradigm for antituberculosis drug scheduling. Antimicrob Agents Chemother 55: 5085-5089.

Sung JC, Garcia-Contreras L, Verberkmoes JL, Peloquin CA, Elbert KJ, Hickey AJ, Edwards DA. 2009a. Dry powder nitroimidazopyran antibiotic PA-824 aerosol for inhalation. Antimicrob Agents Chemother 53: 1338-1343.

Sung JC, Padilla DJ, Garcia-Contreras L, Verberkmoes JL, Durbin D, Peloquin CA, Elbert KJ, Hickey AJ, Edwards DA. 2009b. Formulation and pharmacokinetics of selfassembled rifampicin nanoparticle systems for pulmonary delivery. Pharm Res 26: 1847-1855.

Vernon A, Burman W, Benator D, Khan A, Bozeman L. 1999. Acquired rifamycin monoresistance in patients with HIV-related tuberculosis treated with once-weekly rifapentine and isoniazid. Tuberculosis trials consortium. Lancet 353: 1843-1847.

Vicini P, van der Graaf PH. 2013. Systems pharmacology for drug discovery and development: Paradigm shift or flash in the pan? Clin Pharmacol Ther 93: 379-381.

Wallis RS, Wang C, Meyer D, Thomas N. 2013. Month 2 culture status and treatment duration as predictors of tuberculosis relapse risk in a meta-regression model. PloS ONE 8: e71116.

Weiner M, Burman W, Vernon A, Benator D, Peloquin CA, Khan A, Weis S, King B, Shah N, Hodge T. 2003. Low isoniazid concentrations and outcome of tuberculosis treatment with once-weekly isoniazid and rifapentine. Am J Respir Crit Care Med 167: 1341-1347.

Weiner M, Benator D, Burman W, Peloquin CA, Khan A, Vernon A, Jones B, Silva-Trigo C, Zhao Z, Hodge T. 2005. Association between acquired rifamycin resistance and the pharmacokinetics of rifabutin and isoniazid among patients with HIV and tuberculosis. Clin Infect Dis 40: $1481-1491$.

Weiner M, Peloquin C, Burman W, Luo C-C, Engle M, Prihoda TJ, Mac Kenzie WR, Bliven-Sizemore E, Johnson JL, Vernon A. 2010. Effects of tuberculosis, race, and human gene SLCO1B1 polymorphisms on rifampin concentrations. Antimicrob Agents Chemother 54: $4192-$ 4200 .

Williamson B, Dooley KE, Zhang Y, Back DJ, Owen A. 2013. Induction of influx and efflux transporters and cytochrome P450 3A4 in primary human hepatocytes by rifampin, rifabutin, and rifapentine. Antimicrob Agents Chemother 57: 6366-6369. 


\section{G. Davies}

Zahoor A, Sharma S, Khuller G. 2005. Inhalable alginate nanoparticles as antitubercular drug carriers against experimental tuberculosis. Int $J$ Antimicrob Agents 26: 298-303.

Zhang Y, Mitchison D. 2003. The curious characteristics of pyrazinamide: A review. Int J Tuberc Lung Dis 7: 6-21.

Zhang L, Sinha V, Forgue ST, Callies S, Ni L, Peck R, Allerheiligen SRB. 2006. Model-based drug development:
The road to quantitative pharmacology. J Pharmacokinet Pharmacodyn 33: 369-393.

Zhu H-J, Patrick KS, Yuan H-J, Wang J-S, Donovan JL, DeVane CL, Malcolm R, Johnson JA, Youngblood GL, Sweet DH, et al. 2008. Two CES1 gene mutations lead to dysfunctional carboxylesterase 1 activity in man: Clinical significance and molecular basis. Am J Hum Genet 82: 1241-1248. 


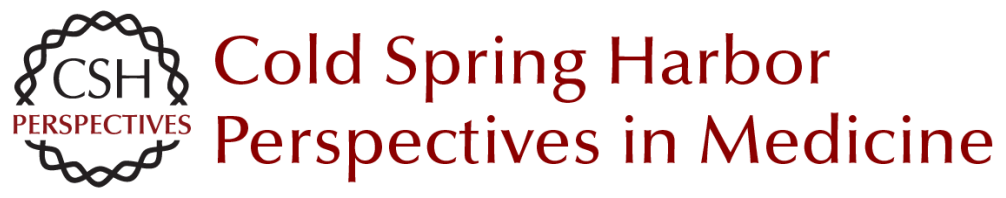

\section{Pharmacologic Considerations in Use and Development of Antituberculosis Drugs}

Geraint Davies

Cold Spring Harb Perspect Med 2015; doi: 10.1101/cshperspect.a021170 originally published online September 18, 2014

\section{Subject Collection Tuberculosis}

\section{Transmission and Institutional Infection Control of Tuberculosis Edward A. Nardell \\ Innate and Adaptive Cellular Immune Responses to Mycobacterium tuberculosis Infection Katrin D. Mayer-Barber and Daniel L. Barber}

Tuberculosis Comorbidity with Communicable and Noncommunicable Diseases

Matthew Bates, Ben J. Marais and Alimuddin Zumla

Host-Directed Therapies for Tuberculosis David M. Tobin

Immunity and Immunopathology in the Tuberculous Granuloma

Antonio J. Pagán and Lalita Ramakrishnan

Tuberculosis Drug Development: History and Evolution of the Mechanism-Based Paradigm? Sumit Chakraborty and Kyu Y. Rhee

\section{Genetic Approaches to Facilitate Antibacterial Drug Development Dirk Schnappinger}

The Tuberculosis Drug Discovery and Development Pipeline and Emerging Drug Targets Khisimuzi Mdluli, Takushi Kaneko and Anna Upton
Clinical Aspects of Adult Tuberculosis

Robert Loddenkemper, Marc Lipman and Alimuddin Zumla

Advances in Diagnostic Assays for Tuberculosis Stephen D. Lawn

Diagnosis and Management of Latent

Tuberculosis Infection

Laura Muñoz, Helen R. Stagg and Ibrahim Abubakar

Mycobacterial Growth Iria Uhía, Kerstin J. Williams, Vahid Shahrezaei, et al.

Multidrug-Resistant Tuberculosis and Extensively

Drug-Resistant Tuberculosis

Kwonjune J. Seung, Salmaan Keshavjee and Michael L. Rich

The Mycobacterial Cell Wall--Peptidoglycan and

Arabinogalactan

Luke J. Alderwick, James Harrison, Georgina S. Lloyd, et al.

Tuberculosis and HIV Coinfection Judith Bruchfeld, Margarida Correia-Neves and Gunilla Källenius

Imaging in Tuberculosis Jamshed B. Bomanji, Narainder Gupta, Parveen Gulati, et al.

For additional articles in this collection, see http://perspectivesinmedicine.cshlp.org/cgi/collection/ 